\title{
BUCHBESPRECHUNGEN
}

\author{
Ralf Wittkowski \\ Die Staatensukzession in völkerrechtliche Verträge unter besonderer Berücksichti- \\ gung der Herstellung der staatlichen Einheit Deutschlands
}

Verlag Peter Lang, Frankfurt a.M. etc., 1992, 401 S.

Die Umwälzungen der Jahre 1989/90 haben das Interesse an einem "ungeliebten Kind" der Völkerrechtslehre neu belebt, dem Recht der Staatensukzession. Spätestens seit Georg Jellinek scheint es zum "guten Ton" zu gehören, dieses Rechtsgebiet als eines der schwierigsten und am wenigstens erforschten zu bezeichnen. Mit Bezug auf die deutsche Vereinigung versucht nun diese bei Blumenwitz in Würzburg entstandene Dissertation wenigstens etwas Licht in das Dunkel zu bringen. Bereits im Februar 1992 abgeschlossen, war sie eine der ersten ausführlichen Stellungnahmen speziell zu den sich aus der deutschen Einheit ergebenden Sukzessionsproblemen. ${ }^{1}$

Die Untersuchung ist in zwei Teile gegliedert, einen allgemein-völkerrechtlichen (S. 6173) und einen besonderen, der sich den konkreten aus der Herstellung der deutschen Einheit entstehenden Fragen der Vertragsnachfolge widmet (S. 174-375).

Der allgemeine Teil beginnt notwendigerweise mit den "Grundlagen der Staatensukzession" (S. 6-46), also mit Begriffsklärungen und Abgrenzungen. Im Mittelpunkt steht hier zunächst der Begriff der Staatensukzession selbst, der in der völkerrechtlichen Literatur schon häufig für Verwirrung gesorgt hat. Wittkowski beschränkt den Begriff zu Recht auf den Gebietswechsel (S. 8-9) und entscheidet sich für das tatbestandsorientierte Verständnis von "Staatensukzession", während er die Rechtsfolgefragen der "Lehre von der Staatensukzession" zuweist (S. 11). Zu begrüßen ist die sich anschließende Abgrenzung der Sukzession von der Staatsidentität (S. 12-14), auch wenn deren Problematik mit dem bloßen Verweis auf die "Drei-Elementen-Lehre" nur sehr unzureichend beschrieben sein dürfte. Nach einer Ausgrenzung der nicht erfaßten Fälle folgt eine Übersicht über die verschiedenen Sukzessionstatbestände (S. 22-28). Dabei fällt auf, daß unter den Fällen des Staatsuntergangs auch noch die Annexion genannt wird (S. 22 unten), während ihr der Autor auf S. 61 mit der heute ganz h.M. jegliche "völkerrechtlichen Wirkungen" abspricht. Zutreffend wird auch der Eintritt in einen Bundesstaat, dem vor allem in der angelsächsischen Lehre häufig ein völkerrechtliches "Eigenleben" nachgesagt wird, in die Sukzessionstatbestände eingereiht (S. 24).

1

Vgl. im übrigen z.B. U. Fastenrath, Die Regelungen über die Staatennachfolge bei der Vereinigung der beiden deutschen Staaten, VRÜ 25 (1992), 67-83; dens., Der deutsche Einigungsvertrag im Lichte des Rechts der Staatennachfolge, AJPIL 44 (1992), 1-54; vorher schon St. Oeter, German Unification and State Succession, ZaöRV 51 (1991), 349-383. 
Die Abgrenzung der Lehre von der Staatensukzession vom vertragsrechtlichen Instrument der "clausula rebus sic stantibus" (S. 36-37) stellt klar, daß eine Ersetzung ersterer durch letztere, wie sie mitunter propagiert wird, unhaltbar ist. Denn in der Regel setzt die vertragsrechtliche Klausel ein fortbestehendes Vertragsverhältnis voraus, so daß die sukzessionsrechtliche Frage der Vertragsnachfolge eine notwendige Vorfrage darstellt. Zum Abschluß dieses ersten Abschnitts stellt der Autor klar, daß er mit der heute ganz überwiegenden Lehre Regeln der Staatensukzession nicht aus abstrakten Formeln ("Universalsukzession", "clean slate") ableitet, sondern nach der induktiven Methode anhand einer Analyse der Staatenpraxis zur konkreten Sukzessionskategorie; Wittkowski nennt dies die "Theorie von der Spezialsukzession" (S. 43-44).

Diesem Ansatz entsprechend folgt ein umfänglicher Abschnitt zur Sichtung der historischen Staatenpraxis (S. 47-158), den der Verfasser nach verschiedenen Vertragskategorien untergliedert (persönliche Verträge - Mitgliedschaften in Internationalen Organisationen - radizierte Verträge), und nur innerhalb dieser Kategorien unterscheidet er jeweils die vorher genannten Sukzessionstatbestände (Zession, Eingliederung, Fusion etc.). M.E. hätte die zuvor beschworene induktive Methode eine umgekehrte Gliederungsweise nahegelegt, da die Möglichkeit einer Ableitung gleichförmiger Praxiselemente bei einer zusammenhängenden Betrachtung der einzelnen Sukzessionskategorien doch größer erscheint. Darüber hinaus ist etwa die Kategorie der "persönlichen Verträge" sehr viel weniger klar umrissen, als es die einzelnen Tatbestände der Staatensukzession sind. Die Unterscheidung in verschiedene Vertragsarten hätte daher am Ende das Ergebnis einer Analyse der Staatenpraxis sein können, als Gliederungsansatz ist sie dagegen kaum verläßlich.

Die Übersicht über die Staatenpraxis selbst muß notwendig kursorisch bleiben, da der zur Verfügung stehende Raum eine umfassende Erörterung aller Sukzessionskategorien nicht erlaubt hätte. Vielleicht wäre auch in diesem Fall "weniger", d.h. die Beschränkung auf den für die deutsche Vereinigung relevanten Tatbestand, die Inkorporation, "mehr" gewesen. So aber behandelt Wittkowski nur die in der gängigen Literatur ohnehin erwähnten Beispielsfälle und vermeidet in streitigen Fällen konkrete Aussagen zur tatbestandlichen Einordnung: So wird zu den Gründungen Italiens, Jugoslawiens und des Deutschen Reiches nur gesagt, daß ihre Einordnung als Inkorporation oder Fusion umstritten gewesen sei (S. 65, 69 bzw. 83); eine eigene Stellungnahme des Autors dazu unterbleibt jedoch, obwohl er erneut hervorhebt, daß diese Unterscheidung "sukzessionsrechtlich von großer Bedeutung" sei (S. 65 und 96). Die jeweils statt dessen gebotene Schilderung der Behandlung der bestehenden völkerrechtlichen Verträge kann eine genaue tatbestandliche Einordnung nicht wirklich ersetzen. Auch die kommentarlose Einordnung des "Anschlusses" Österreichts 1938 unter die Eingliederungsfälle (S. 69-70) ist unbefriedigend; zumindest ein kurzes Eingehen auf die Stimmen, die diese Sukzession als "nul et non avenu" ansehen, wäre hier angebracht gewesen. Die Behandlung der Auflösung von Realunionen als Fälle der Dismembration (S. 103-105, 109) ist ebenfalls irritierend, da es sich dabei um 
die Beendigung von Staatenverbindungen, also gerade nicht um den Zerfall eines Staates handelt.

Die Lektüre dieses Abschnitts wird dadurch erleichtert, daß Wittkowski zu jeder Sukzessionskategorie eine knappe Auswertung der Staatenpraxis gibt. Leider wird der Ertrag für die völkerrechtspraktische Nutzanwendung etwas entwertet, wenn er in der Zusammenfassung am Ende des Praxisüberblicks (S. 155-158) nicht zwischen bindenden völkerrechtlichen Regeln und bloßen "Tendenzen" unterscheidet.

Der letzte Abschnitt des allgemeinen Teils ist der Wiener Konvention über die Staatennachfolge in Verträge von 1978 gewidmet (S. 159-173), deren Inhalt zunächst kurz dargestellt und anschließend einer Kritik unterzogen wird. Der Haupteinwand, daß die Konventionsregeln die Ergebnisse der historischen Staatenpraxis nur sehr unvollkommen wiedergeben, bringt Wittkowski zu der Prognose, daß sich die für ein Inkrafttreten erforderliche Zahl von 15 Vertragsstaaten "nur schwerlich finden lassen (wird)". Allerdings hatte sich die von ihm - anhand von Sekundärzitaten - angegebene Zahl von 8 Ratifikationen bzw. Beitritten zum 31.12.1992 bereits auf 12 erhöht. $^{2}$

Der zweite Teil der Arbeit, der sich den konkreten durch die Herstellung der Einheit Deutschlands aufgeworfenen Fragen der Staatennachfolge in Verträge zuwendet, beginnt mit einer Darstellung der Rechtslage Deutschlands vor und nach dem 3.10.1990 (S. 174196). Nachdem Wittkowski die ganz herrschende westliche Auffassung von der Identität der Bundesrepublik Deutschland mit dem Deutschen Reich und dem erfolglosen Sezessionsversuch der DDR rekapituliert hat, subsumiert er den Beitritt der DDR gemäß Art. 23 S. 2 GG a.F. unter die im 1. Teil erörterten völkerrechtlichen Kategorien und kommt zutreffend zu der - kaum überraschenden - Einordnung als Inkorporation (S. $184 \mathrm{f}$.). Richtig ist auch der in einem anschließenden Exkurs (S. 185-189) gemachte Hinweis, daß auch die Herbeiführung der deutschen Einheit über den Weg des Art. 146 GG a.F. die völkerrechtliche Klassifizierung der staatlichen Vereinigung als Eingliederung nicht nowendig ausgeschlossen hätte.

Es folgt ein Kapitel über das Schicksal der völkerrechtlichen Verträge der Bundesrepublik Deutschland (S. 197-271), das zu Recht von deren Fortbestand und Ausdehnung auf das eingegliederte Gebiet ausgeht und diese Rechtsfolge dem Völkergewohnheitsrecht entnimmt, das durch Art. 11 EV nur bestätigt wurde. Leider erliegt auch dieser Verfasser dem herrschenden Trend, diese Rechtsfolge der Inkorporation mit dem Etikett vom "Grundsatz der beweglichen Vertragsgrenzen" zu belegen (S. 198 u.ö.), obwohl dieser herkömmlich (nur) auf den Tatbestand der Zession gemünzt ist und wegen des Plurals schon terminologisch auch nur dort paßt - nur bei der identitätserhaltenden Gebietsabtretung verschieben sich eben mehrere "Vertragsgrenzen", während bei der Eingliederung allein die "Vertragsgrenze" des inkorporierenden Staates "beweglich" ist. 
In einem Unterabschnitt wendet sich Wittkowski sodann den Besonderheiten bei der Herstellung der deutschen Einheit sowie den speziellen Problemen des "2+4"-Vertrages zu (S. 205-242). Seine hierbei vertretene Ansicht, die Bundesrepublik Deutschland habe aufgrund eines "Legitimationsdefizits" bis zum 3.10.1990 keine völkerrechtlichen Bindungen für den fortbestehenden deutschen Gesamtstaat erzeugen können (S. 206-207), ist mit der zuvor rezitierten Identitätsthese, wonach die Bundesrepublik eben gerade dieser fortbestehende Gesamtstaat ist, schlichtweg unvereinbar - und im übrigen für den Kontext der Staatensukzession auch vollkommen überflüssig. Konsequente Verfechter der Identitätstheorie des Bundesverfassungsgerichts müßten die Ausdehnung der völkerrechtlichen Verträge der Bundesrepublik statt auf die autonomen Regeln der Staatensukzession auf die mit dem 3.10.1990 möglich gewordene Erstreckung ihrer Hoheitsgewalt auf das gesamte Staatsgebiet stützen - eine Lösung, die Wittkowski selbst weiter unten für das Reichskonkordat vertritt (S. 282).

Anschließend geht der Autor auf das Schicksal einzelner Verträge der Bundesrepublik Deutschland ein (S. 243-271). Daß ihre Mitgliedschaft in den Vereinten Nationen unberührt bleibt (S. 243), ist angesichts ihrer Subjektsidentität mit dem vereinten Deutschland selbstverständlich. Der Hinweis auf die bekannte Stellungnahme des Rechtsausschusses der UN-Generalversammlung von 1947 ist in diesem Zusammenhang eher verwirrend, da es dort um einen anders gelagerten Fall ging, nämlich um die Abspaltung Pakistans von Indien und ihre Auswirkung auf die indische Mitgliedschaft. Schließlich ist die Aussage, die Feindstaatenklauseln der UN-Charta seien seit dem UN-Beitritt der beiden deutschen Staaten "überholt und nicht mehr anwendbar" (S. 243), zumindest ungenau; in einer völkerrechtlichen Doktorarbeit hätte man sich wenigstens einen kurzen Hinweis auf die dogmatische Begründung gewünscht.

Bei der Behandlung des Londoner Schuldenabkommens (LSA) von 1953 geht Wittkowski insbesondere auf das "Reparations-Moratorium" des Art. 5 Abs. 2 und die Entschädigungsforderungen ehemaliger polnischer Zwangsarbeiter ein (S. 246-249). Er konstruiert eine Bindung des Nichtvertragsstaates Polen an die genannte Vorschrift mit Hilfe ihrer gewohnheitsrechtlichen Geltung. Diese Behauptung wird nicht nur nicht belegt und dürfte angesichts der quellentheoretischen Voraussetzungen von Völkergewohnheitsrecht auch kaum zu belegen sein (Stichworte: Staatenpraxis und Rechtsüberzeugung neben dem Vertrag? Polen als "persistent objector"?). Eine solche Konstruktion ist auch vollkommen überflüssig, da Polen 1953 einseitig (auch) auf mögliche Entschädigungsforderungen seiner Staatsangehörigen verzichtet hat. ${ }^{3}$ Für die Forderungen ehemaliger Zwangsarbeiter aus Ungarn und Rumänien zieht die h.M. im übrigen Art. 5 Abs. 4 LSA heran, der aus

3

Vgl. dazu A. Randelzhofer / O. Dörr, Entschädigung für Zwangsarbeit? Zum Problem individueller Entschädigungsansprüche von ausländischen Zwangsarbeitern während des Zweiten Weltkrieges gegen die Bundesrepublik Deutschland (1994), S. 69-74. 
Gründen seines personellen und sachlichen Anwendungsbereichs diese Fälle jedoch ebensowenig regelt. ${ }^{4}$

Zum Abschluß dieses Kapitels behandelt der Autor die EG-Mitgliedschaft des vereinten Deutschlands, deren Fortbestehen er aus dem allgemeinen Völkerrecht der Staatensukzession folgert (S. 255-262), sowie die Ostverträge von 1970/73 (S. 262-268). Im Hinblick auf letztere macht sich bemerkbar, daß die Untersuchung offenbar doch einige Zeit vor dem Jahresende 1991 abgeschlossen wurde, da für einen "Zerfall" der Sowjetunion im Text nur Ereignisse im September 1991 erörtert werden, während dieser Fall einer Staatensukzession erst im Dezember jenes Jahres richtig relevant wurde (vgl. die Anmerkung auf S. 268).

Es folgt ein kurzer Abschnitt über die noch bestehenden völkerrechtlichen Verträge des Deutschen Reiches (S. 272-283), deren andauernde Bindungskraft Wittkowski zutreffend mit der Subjektsidentität - nicht etwa: Rechtsnachfolge! - der Bundesrepublik Deutschland begründet.

Der Kern der sukzessionsrechtlichen Problematik der Inkorporation betrifft stets die Verträge des eingegliederten Staates, so daß dem Schicksal der für die DDR bestehenden völkerrechtlichen Bindungen zu Recht ein breiter Raum eingeräumt wird (S. 284-269). Da er den konkreten Regeln des deutschen "Einigungsrechts" insoweit keine verbindlichen Vorgaben entnehmen kann (S. 284-302), untersucht der Verfasser die Frage anhand des allgemeinen Völkerrechts (S. 303-310). Die dabei aus der im 1. Teil aufgeführten Staatenpraxis deduzierte Essenz erschöpft sich jedoch im Erlöschen der sog. persönlichen und im Fortbestand der sog. radizierten Verträge (S. 304). Dieses war im wesentlichen bereits vor der deutschen Vereinigung der Stand der völkerrechtlichen Erkenntnis ${ }^{5}$ und ist daher als Ertrag einer speziell mit diesem Komplex befaßten Dissertation ein wenig enttäuschend. Denn interessant wäre gerade eine Differenzierung nach den Vertragsarten, die sich in der Mehrzahl zwischen den beiden Polen "hochpolitisch" und "radiziert" bewegen. Immerhin ist es hilfreich, daß der Verfasser eine Auswahl fortbestehender, weil radizierter DDR-Verträge nennt (S. 308-310).

Auch im Hinblick auf die DDR-Verträge ist wieder ein eigener Abschnitt den Besonderheiten der Herstellung der deutschen Einheit gewidmet (S. 310-332), zu denen Wittkowski auch den "Gesichtspunkt des Vertrauensschutzes" zählt (S. 321-327). Ein letztes Kapitel geht exemplarisch auf "wichtige völkerrechtliche Verträge der DDR" ein (S. 332369), von denen zunächst die im Rahmen des RGW bestehenden Bindungen problematisiert werden. Der Verfasser plädiert hier grundsätzlich für eine analoge Anwendung des Art. 234 EWGV (jetzt: EGV) auf die Rechtsnachfolge durch Staatensukzession in Verträge mit Drittstaaten (S. 338-339). Angesichts der fundamentalen Unterschiede zwischen dem Beitritt zur Gemeinschaft und der bloßen Gebietsvergrößerung eines Mitgliedstaates ist allerdings zweif elhaft, ob die Voraussetzungen einer Analogie wirklich vorliegen. Im

4

Dazu Randelzhofer / Dörr, a.a.O., S. 96-98.

5 Vgl. nur J.A. Frowein, VVDStRL 49 (1990), 26 f.; K. Hailbronner, JZ 1990, 449 (453 f.). 
übrigen ist zu erwägen, ob nicht, wenn man - wie Wittkowski - die Vereinbarkeit mit der inkorporierenden Rechts- und Wirtschaftsordnung als Kriterium für die Entscheidung über Fortgeltung oder Erlöschen eines völkerrechtlichen Vertrages ansieht, dann auch die Vorschriften des Gemeinschaftsrechts zur Rechtsordnung der Bundesrepublik Deutschland zu zählen sind - mit der Folge, daß sich das Problem der Anpassungsverpflichtung gemäß Art. 234 S. 2 EGV überhaupt nicht stellt. Die Abkommen der DDR zur Regelung der doppelten Staatsangehörigkeit sieht der Verfasser wohl zutreffend als erloschen an, auch wenn er etwas vollmundig behauptet, eine von der deutschen Staatsangehörigkeit verschiedene DDR-Staatsbürgerschaft habe "es nie gegeben" (S. 355). Das Erlöschen der kollisionsrechtlichen Staatsverträge der DDR wird u.a. damit begründet, daß es ansonsten zu Kollisionen mit Verträgen der Bundesrepublik käme und im übrigen fraglich wäre, ob sich die Fortgeltung nur auf das Beitrittsgebiet oder aber das ganze Bundesgebiet bezieht (S. 368). Nicht nur jedoch sind das Entstehen schwieriger Rechtsfragen oder von "Rechtsanwendungsproblemen" kaum überzeugende Argumente im Streit um dogmatische Positionen, auch treffen diese Erwägungen auf jeden Fall der "nicht radizierten Fortgeltung" zu und sind, bezogen auf eine bestimmte Vertragsart, daher schwer nachvollziehbar. Eine Zusammenfassung (S. 376-394) rundet die Darstellung ab. Im Anhang finden sich Auszüge aus der - nicht in Kraft befindlichen - Konvention über die Staatensukzession in Verträge (S. 395-401), die allerdings nicht aus den Originaldokumenten, sondern aus der deutschen Übersetzung bei Poeggel/Meissner/Poeggel zitiert werden.

Insgesamt handelt es sich um eine anregende Arbeit, die die Bedeutung des Rechts der Staatensukzession erneut in den Blickpunkt rückt und die Probleme dieses Rechtsgebiets systematisch aufbereitet. An den eingangs erwähnten Unklarheiten - im Hinblick sowohl auf dogmatische Verwerfungen als auch auf die uneinheitliche Staatenpraxis - ändert jedoch auch diese Untersuchung leider nicht viel. Der Leser wird den Eindruck nicht los, daß hier "mehr drin" gewesen wäre. Die wesentlichen Ergebnisse der Arbeit finden sich im übrigen auch in einer nur kurz danach erschiedenen Monographie von Blumenwitz. ${ }^{6}$

Oliver Dörr

\section{The Earth Summit: The United Nations Conference on Environment and Develop-} ment (UNCED). Introduction and Commentary by Stanley P. Johnson, International Environmental Law and Policy Series, Graham \& Trotman / Martinus Nijhoff, London / Dordrecht / Boston, 532 pp., US \$140.00

Im Juni 1992 fand die United Nations Conference on Environment and Development (UNCED) in Rio de Janeiro statt, 20 Jahre nach der Stockholmer Umweltkonferenz der

D. Blumenwitz, Staatennachfolge und die Einigung Deutschlands, Teil I: Völkerrechtliche Verträge (1992); dazu der Teil II (Staatsvermögen und Staatsschulden) von G. Gornig. 